\title{
Ventilación mecánica no invasiva en UCI en paciente con insuficiencia respiratoria como forma de presentación de dermatomiositis
}

\author{
B. OBÓN AZUARA, I. GUTIÉRREZ CÍA, C. SÁNCHEZ POLO \\ Servicio de Medicina Intensiva. Hospital Obispo Polanco. Teruel
}

\begin{abstract}
RESUMEN
La polimiosistis y dermatomiositis son miopatías inflamatorias idiopáticas con frecuentes complicaciones respiratorias aunque infrecuente fallo respiratorio agudo, que tiene lugar en las formas de presentación más severas y precisa soporte ventilatorio. Presentamos el caso de una paciente diagnosticada de dermatomiositis (DM) que es ingresada en UCI debido a fallo respiratorio secundario a debilidad de los músculos respiratorios con pobre respuesta inicial a terapia corticoidea -con prednisolona en pulsos- e inmunosupresora con metotrexate, y posteriormente ciclofosfamida y ciclosporina. Una vez realizado su ingreso en UCI recibe tratamiento con ventilación mecánica no invasiva con éxito, asî como añadiendo altas dosis de inmunoglobulinas intravenosas que aunque de empleo controvertido, se reveló como único tratamiento posible dada la refractariedad de la enfermedad frente a la inmunosupresión.
\end{abstract}

PALABRAS CLAVE: Dermatomiositis y UCI. Medicina Intensiva y ventilación mecánica no invasiva. Insuficiencia respiratoria y dermatomiositis. Inmunoglubulina intravenosa.
NON INVASIVE MECHANICAL VENTILATION IN INTENSIVE CARE UNIT IN PATIENT WITH RESPIRATORY FAILURE AS THE FIRST MANIFESTATION OF DERMATOMYOSITIS

Obón Azuara B, Gutiérrez Cía I, Sánchez Polo C. Ventilación mecánica no invasiva en UCI en paciente con insuficiencia respiratoria como forma de presentación de dermatomiositis. An Med Interna (Madrid) 2005; 22: 434-436.

\section{INTRODUCCIÓN}

Las enfermedades musculares inflamatorias representan un grupo heterogéneo de patologías con diferente patogénesis, caracterizado por la debilidad de los músculos proximales. La polimiosistis y dermatomiositis son miopatías inflamatorias idiopáticas en las que si bien son frecuentes las complicaciones respiratorias, registrándose una incidencia de hasta el $45 \%$, no lo es el fallo respiratorio agudo, que tiene lugar en las formas de presentación más severas y precisa ocasionalmente soporte ventilatorio e incluso ingreso en las unidades de cuidados intensivos (1-5).

\begin{abstract}
Polymiositis and dermatomyositis are idiopathic inflammatory myositis with several respiratory complications but the acute failure respiratory is uncommon, and in the more severity types needs noninvasive mechanical ventilation. The case report a dermatomyositis patient who needed intensive care to failure respiratory due to muscle weakness who did not respond to corticoids - with pulsed prednisolone- in combination with another immunosuppressive agent with methotrexate, and after this with cyclofosfamide and cyclosporin. Along her stay in ICU is used a succesfully treatment with noninvasive mechanical ventilation and added high dose immunoglobulin intravenous, although there is a controversial about its use, was an effective treatment due to drug resistant dermatomyositis front to immunosuppression.
\end{abstract}

KEY WORDS: Dermatomyositis and ICU. Intensive Care Medicine and non invasive mechanical ventilation. Respiratory failure and dermatomyositis. Intravenous immunoglobulin. 
subir escaleras y levantar objetos. Hace 2 meses, tras cuadro febril de $39{ }^{\circ} \mathrm{C}$ que remitió espontáneamente, presentó un episodio diarreico seguido de lesiones descamativas palmoplantares tipificadas como lesiones compatibles con dermatitis eczematosa, recibiendo tratamiento con esteroides tópicos por este motivo. En las últimas $48 \mathrm{~h}$ presenta además disfagia y edema facial.

A su ingreso en el hospital en la exploración física se aprecia edema facial, lesiones eritemato descamativas a nivel perioral y nasal y articulaciones metacarpofalángicas. No muestra fenómeno de Raynaud, ni fenómenos de fotosensibilidad, artritis o artralgias. No refiere ingesta ni exposición a tóxicos. Además existe una disminución de la fuerza en las extremidades superiores e inferiores con preservación de los reflejos osteotendinosos. El resto de la exploración física inicial es compatible con la normalidad.

\section{EXPLORACIONES COMPLEMENTARIAS}

Los primeros análisis de sangre muestran unos valores de enzimas musculares elevados: LDH 4296; CPK 9286; aldolasa 227; otros parámetros: AST 418; ALT 273, siendo el resto de las determinaciones normales.

Ante la sospecha de enfermedad muscular se realizan más exámenes complementarios, en los que se descarta inicialmente la etiología infecciosa del proceso con serologías a Salmonella tiphy $\mathrm{H}$ y O, Brucella, Toxoplasma, TPHA, VE-B, CMV, Yersinia, hidatidosis, Micoplasma, VHS negativos todos ellas, y títulos de IgM anti CMV 0,52, Ig G antiVHC y VHB inferiores a 0,10 (compatibles con la normalidad).

Simultáneamente se tienen en cuenta otras etiologías como la neoplásica, con extracción de marcadores tumorales negativos (CA 12-5: $12 \mathrm{u} / \mathrm{ml}$; CA 15.3: 14,61 u/ml; CA 19-9: 5,62 u/ml), así como la inmunológica, que únicamente muestra valores positivos para los ANA, siendo el resto normales: ANA positivo 1/2560; anti DNA negativo; Ac anti Mi-1/Mi-2 y anti SRP con títulos inferiores a 1:160, anti ENA (RNP/Sm) ambos inferiores a 0,10 y Ac antiJo-1 inferior a 0,10. cANCA 1,44 y pANCA 2,04 (valores normales).

También se toma en consideración la etiología inflamatoria, y así se realiza una biopsia muscular y un estudio neurofisiológico con resultados compatibles con cuadro miopático con importantes signos inflamatorios. Biopsia cuádriceps: importante infiltrado inflamatorio linfocitario de forma parcheada de distribución perivascular perimisial y endomisial, con fenómenos de necrosis de fibras musculares y signos de atrofia perifascicular. No presenta fibras hipertrofiadas ni fibrosis endomisial. EMG/ENG en ambos deltoides y cuádriceps con intensa actividad de denervación aguda acompañada de potenciales de unidad motora de baja amplitud, duración disminuida y alta incidencia de polifásicos.

Así mismo se descarta la afectación cardiaca mediante la realización de ecocardiograma y registro Holter que no muestran alteraciones. Y se realiza un estudio funcional pulmonar compatible con patrón restrictivo (FVC 0,96 litros, FV1 0,94, FV1/FVC 98, sin qye se pueda realizar el estudio de DLCO ni TCL dada la situación de la paciente).

Ante la evolución clínica compatible con una insuficiencia respiratoria y debido a los hallazgos encontrados, se realiza una TAC pulmonar que muestra un patrón en vidrio deslustrado que afecta a la totalidad de ambos pulmones con áreas de mayor densidad en ambas bases, y presencia de patrón reticular fino bilateral y difuso (Fig. 1).

Durante la evolución clínica existe una persistencia e incremento de la debilidad muscular en ambas cinturas escapular y pélvica, por lo que se inicia tratamiento con metilprednisolona administrada a pulsos cada 24 horas desde el primer momento. Sin embargo, ante la severidad del cuadro clínico al que se asocia una disfagia importante, se debe añadir tratamiento con imnumosupresión múltiple, comenzando inicialmente con metotrexate solo, y cambiando a ciclofosfamida a dosis de $750 \mathrm{mg} / 24 \mathrm{~h}$ acompañada de ciclosporina a dosis de

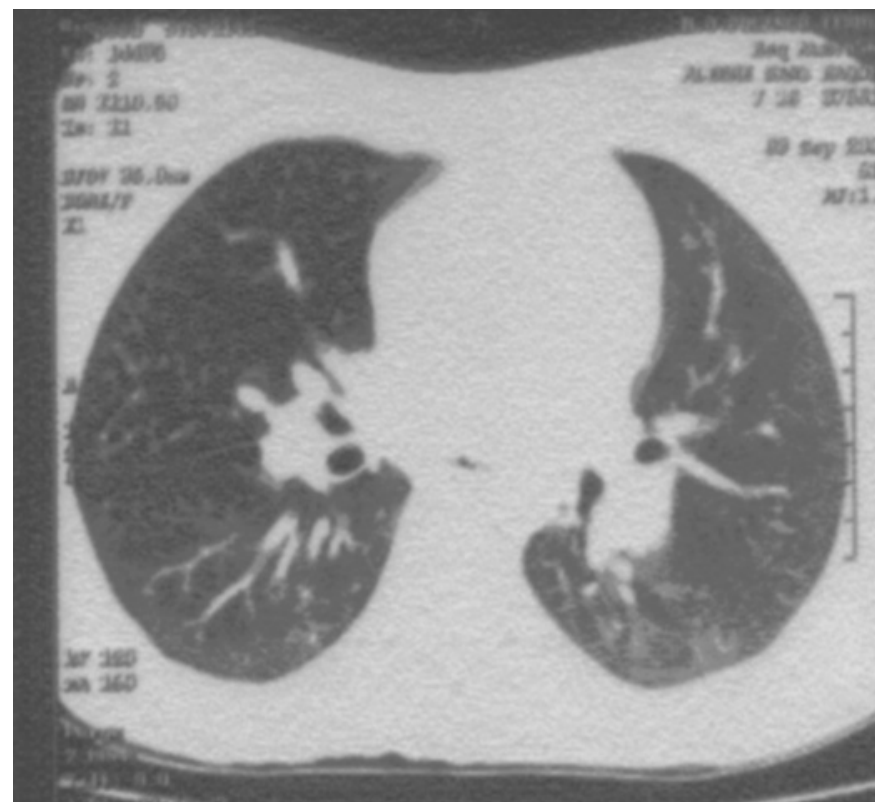

Fig. 1. TAC pulmonar: patrón de vidrio deslustrado que afecta a ambos pulmones.

$150 \mathrm{mg} / 12 \mathrm{~h}$, ambas por vía oral. Pese a ello desarrolla insuficiencia respiratoria acompañada de síndrome febril (con patrón radiológico mostrando ausencia de condensaciones), que empeora progresiva y rápidamente con la siguiente gasometría arterial realizada con fuente de oxigeno al $40 \%$ ( $\mathrm{pH} 7,3 \mathrm{CO} 265,8 \mathrm{O}_{2} 58, \mathrm{SaO}_{2} 86 \%$ ) por lo que es ingresada en la UCI. En el Servicio de Medicina Intensiva se aprecia una marcada hipoventilación global con una saturación de oxigeno obtenida mediante pulsioximetría del $84 \%$, por lo que se inicia tratamiento con BIPAP con altas presiones espiratorias y elevadas FiO2. Tras implantar tratamiento con antibioterapia intravenosa y profilaxis para Pneumocistis carinii, se añade al tratamiento inmunosupresor mencionado inmunoglobulinas intravenosas a dosis de 2 $\mathrm{g} / \mathrm{kg} /$ día, y se implanta una sonda nasogástrica por la que se inicia nutrición enteral a las 24 horas de su ingreso. Tras 12 días de tratamiento inmunosupresor asociado a inmunoglobulinas y a ventilación mecánica no invasiva, la paciente pudo ser dada de alta a planta donde prosiguió con doble inmunosupresión e inmunoglobulinas IV a altas dosis, encontrándose en la actualidad en su domicilio.

\section{DISCUSIÓN}

La ausencia de historia familiar de miopatía, el sexo, la edad de presentación, las características típicas del electromiograma, y posteriormente los hallazgos en la anatomía patológica dejaban pocas dudas acerca del diagnóstico: se trataba de una dermatomiositis que debutó con un compromiso de la musculatura proximal de cintura escapular y pelviana, instaurando tratamiento con corticoides y asociando posteriormente inmunosupresores.

Aunque la afectación de la musculatura respiratoria puede ocurrir en el transcurso de la enfermedad (1-3), y conociendo que entre los factores que empobrecen su pronóstico se encuentra precisamente la participación pulmonar $(7,8)$, lo que no resulta frecuente es que dicha afectación irrumpa de una forma tan severa que requiera el ingreso en Unidades de Cuidados Intensivos, y así, algunos autores proponen que cuando el tratamiento inicial con corticoides e inmunosupre- 
sores no es eficaz, se encuentra indicado el ingreso de estos pacientes en la UCI para llevar a cabo un adecuado y monitorizado soporte ventilatorio (4-6).

En el caso presentado, en el Servicio de Medicina Intensiva se llevó a cabo una ventiloterapia en régimen no invasivo que afortunadamente fue tolerada con éxito por la paciente, que unida a la optimización de los requerimientos nutriciona-

\section{Bibliografía}

1. Bohan A, Peter JB. Polimyositis and dermatomyositis (parts 1 and 2). $\mathrm{N}$ Engl J Med 1975; 292: 344-7.

2. Griggs RC, Askanas V, Di Mauro S, Engel A, et al. Inclusion body myositis and miopathies. Ann Neurol 1995; 38:705-12.

3. Mastaglia FL, Philipps BA, Zirko P. Treatment of inflammatory myopaties. Muscle Nerve 1997; 20: 651-64.

4. Selva-O’Callahan A, Sánchez Sitjes L, Muñoz Gal X, et al. Respiratoy failure due to muscle weakness in inflammatory myopaties: manteninance therapy with home mechanical ventilation. Rheumatology 2000; 39: 914-6.

5. Selva A, Cuenca R, Sanjosé A, Navarro C, et al. Pulmonary involvement in PM/DM. Br J Rheumatol 1990; 29: 111.

6. Arakawa H, Yamada H, Kurihara Y, et al. Nonspecific intersitial pneumonia associated with polymiositis and dermatomyositis. Chest 2003; 123: 1096-103. les, así como a la utilización de inmunoglobulina intravenosa a altas dosis, ante la escasa respuesta inicial a la inmunosupresión, y de eficacia no probada en todos los estudios, aunque descrita como efectiva en los pacientes con dermatomiositis y polimiositis refractarias a inmunosupresión como tercera línea de terapia (9-11), se mostraron como factores decisivos para lograr la supervivencia de la paciente.

7. Choy EHS, Isenberg DA. Treatment of dermatomyositis and polymyositis. Rheumatology 2002; 41: 7-13.

8. Rockall AG, Rickards D. Imaging of the pulmonary manifestations of systemic disease. BJM 2001; 77 (912): 621-38.

9. Mastaglia FL, Phillips BA, Zilko PJ. Immunoglobulin therapy in inflammatory myopathies. J Neurol Neurosurg Psychiatry 1998; 65: 107-10.

10. Dalakas MC. The use of intravenous immunoglobulin in the treatment of autoinmune neuromuscular diseases: evidence-based indication and safety profile. Pharmacol Ther 2004; 102 (3): $177-$ 93.

11. Pyne D, Ehrenstein M, Morris V. The therapeutic uses of intravenous immunoglobulins in autoimmune rheumatic diseases. Rheumatology 2002; 41: 367-74. 